Article

\title{
A Mixed Finite Volume Element Method for Time-Fractional Reaction-Diffusion Equations on Triangular Grids
}

\author{
Jie Zhao ${ }^{1,2}$, Hong Li ${ }^{1, *(\mathbb{D})}$, Zhichao Fang ${ }^{1, *(\mathbb{D})}$ and Yang Liu ${ }^{1}$ \\ 1 School of Mathematical Sciences, Inner Mongolia University, Hohhot 010021, China \\ 2 School of Statistics and Mathematics, Inner Mongolia University of Finance and Economics, \\ Hohhot 010070, China \\ * Correspondence: smslh@imu.edu.cn (H.L.); mazcfang@aliyun.com (Z.F.)
}

Received: 31 May 2019; Accepted: 2 July 2019; Published: 5 July 2019

check for updates

\begin{abstract}
In this article, the time-fractional reaction-diffusion equations are solved by using a mixed finite volume element (MFVE) method and the L1-formula of approximating the Caputo fractional derivative. The existence, uniqueness and unconditional stability analysis for the fully discrete MFVE scheme are given. A priori error estimates for the scalar unknown variable (in $L^{2}(\Omega)$-norm) and the vector-valued auxiliary variable (in $\left(L^{2}(\Omega)\right)^{2}$-norm and $\boldsymbol{H}(\operatorname{div}, \Omega)$-norm) are derived. Finally, two numerical examples in one-dimensional and two-dimensional spatial regions are given to examine the feasibility and effectiveness.
\end{abstract}

Keywords: mixed finite volume element method; time-fractional reaction-diffusion equation; existence and uniqueness analysis; unconditional stability analysis; a priori error estimate

AMS Subject Classification: 65M08; 65M15; 65M60

\section{Introduction}

Fractional partial differential equations (FPDEs) have received more and more attention from scholars in the fields of science and engineering because of their various practical applications [1-6]. Therefore, theoretical research and some problems of the solutions of FPDEs have been closely studied by many scholars [7-11]. However, there are still many FPDEs for which it is difficult to obtain analytic solutions. Thus, a lot of numerical techniques have been constructed and studied to solve FPDEs [12-22], including finite difference (FD) methods, finite element (FE) methods, mixed finite element (MFE) methods, spectral methods, (local) discontinuous Galerkin (L)DG methods and so on.

In recent years, finite volume (FV) and finite volume element (FVE) methods have been used to solve FPDEs by many scholars. Wang and Du [23] constructed a fast locally conservative FV method to solve steady-state space-fractional diffusion equations with Riesz fractional derivatives. Hejazi et al. [24,25] provided an FV method for the time-space two-sided fractional advection-dispersion equation and space fractional advection-dispersion equation, respectively. Zhuang et al. [26] proposed FV and FE methods for the space-fractional Boussinesq equation with Riemann-Liouville derivatives on a one-dimensional domain. Yang et al. [27] constructed an FV scheme with pretreatment Lanczos method to solve two-dimensional space fractional reaction-diffusion equations. Cheng et al. [28] provided an Eulerian-Lagrangian control volume method to solve the space-fractional advection-diffusion equations with Caputo fractional derivatives. Feng et al. [29] proposed an FV scheme to treat the two-sided space-fractional diffusion equation with Riemann-Liouville fractional derivatives. Jia and Wang [30,31] constructed fast FV schemes 
for two kinds of space-fractional differential equations, respectively. Simmons et al. [32] proposed an FV method to solve two-sided fractional diffusion equations with Riemann-Liouville derivatives. Jiang and $\mathrm{Xu}$ [33] constructed a monotone FV scheme to solve the time-fractional Fokker-Planck equation. Karaa et al. [34] proposed an FVE method to solve two-dimensional fractional subdiffusion problems with the Riemann-Liouville derivative. Karaa and Pani [35] applied an FVE method to solve the time-fractional diffusion equations with nonsmooth initial data.

In this article, we will develop a mixed finite volume element (MFVE) method to solve the time-fractional reaction-diffusion equations as follows

$$
\begin{cases}\frac{\partial^{\alpha} u(X, t)}{\partial t^{\alpha}}-\varepsilon \Delta u(X, t)+p(X) u(X, t)=f(X, t), & (X, t) \in \Omega \times J \\ u(X, t)=0, & (X, t) \in \partial \Omega \times \bar{J} \\ u(X, 0)=u_{0}(X), & X \in \bar{\Omega},\end{cases}
$$

where $\Omega \subset R^{2}$ is a bounded convex polygonal domain with boundary $\partial \Omega, J=(0, T]$ with $0<T<\infty$. The functions $u_{0}(X), p(X)$ and $f(X, t)$ are smooth enough, the diffusion coefficient $\varepsilon>0$. And there exist constants $p_{0}>0$ and $p_{1}>0$ such that $0<p_{0} \leq p(X) \leq p_{1}, \forall X \in \bar{\Omega}$. $\frac{\partial^{\alpha} u(X, t)}{\partial t^{\alpha}}$ is the Caputo fractional derivative defined by

$$
\frac{\partial^{\alpha} u(X, t)}{\partial t^{\alpha}}=\frac{1}{\Gamma(1-\alpha)} \int_{0}^{t} \frac{\partial u(X, s)}{\partial s} \frac{\mathrm{d} s}{(t-s)^{\alpha}}, 0<\alpha<1 .
$$

Our aim is to construct an MFVE scheme [36-41] by combining the MFE method [42-45] with the FVE method [46-51] to treat the time Caputo fractional reaction-diffusion equation. The MFVE method can not only simultaneously compute several different quantities but also maintain the local conservativity, which is very important in computational fluid dynamics. In this article, we apply the L1-formula [52,53] to approximate the Caputo fractional derivative, introduce a vector-valued auxiliary variable to rewrite the model equation as the first-order coupled system, and approximate this coupled system by the MFVE method. We use the lowest order Raviart-Thomas $\left(R T_{0}\right)$ space and the piecewise constant function space as the trial function spaces of the vector-valued auxiliary variable and scalar unknown variable, respectively. In terms of theoretical analysis, we give the existence, uniqueness and stability analysis, and obtain a priori error estimates for the scalar unknown variable (in $L^{2}(\Omega)$-norm) and the vector-valued auxiliary variable (in $\left(L^{2}(\Omega)\right)^{2}$-norm and $\boldsymbol{H}(\operatorname{div}, \Omega)$-norm). Further, we give two numerical examples in one-dimensional and two-dimensional spatial regions to verify the feasibility and effectiveness of the proposed scheme.

The remaining structure of the article is as follows. In Section 2, we present a fully discrete MFVE scheme for the time-fractional reaction-diffusion equation by introducing transfer operator $\gamma_{h}$ and the L1-formula of approximating the Caputo fractional derivative. In Section 3, the properties of transfer operator and truncation errors of L1-formula are given, and the generalized MFVE projection and its estimates are introduced. The existence, uniqueness, stability and convergence analysis for the MFVE scheme are analyzed in Sections 4 and 5, respectively. Two numerical examples are given to verify the feasibility and effectiveness in Section 6. Throughout the article, we use the standard notations and definitions of the Sobolev spaces as in Reference [54]. The mark $C$ will denote a generic positive constant which is independent of the spatial mesh parameter $h$ and time discretization parameter $\tau$.

\section{Fully Discrete MFVE Scheme}

In order to formulate the MFVE approximate scheme, we introduce an auxiliary variable $\sigma(X, t)=-\nabla u(X, t)$, and rewrite the problem (1) as

$$
\begin{cases}\text { (a) } & \sigma(X, t)+\nabla u(X, t)=0 \\ \text { (b) } & \frac{\partial^{\alpha} u(X, t)}{\partial t^{\alpha}}+\varepsilon \operatorname{div} \sigma(X, t)+p(X) u(X, t)=f(X, t) .\end{cases}
$$


The associated mixed variational formulation of (3) is to find $\{u, \sigma\}:[0, T] \rightarrow L^{2}(\Omega) \times \boldsymbol{H}(\operatorname{div}, \Omega)$ such that

$$
\left\{\begin{array}{lll}
(a) & (\boldsymbol{\sigma}, \mathbf{w})-(u, \operatorname{div} \boldsymbol{w})=0, & \forall \boldsymbol{w} \in \boldsymbol{H}(\operatorname{div}, \Omega) \\
(b) & \left(\frac{\partial^{\alpha} u}{\partial t^{\alpha}}, v\right)+\varepsilon(\operatorname{div} \sigma, v)+(p u, v)=(f, v), & \forall v \in L^{2}(\Omega), \\
(c) & u(X, 0)=u_{0}(X), \sigma(X, 0)=-\nabla u_{0}(X), & \forall X \in \bar{\Omega},
\end{array}\right.
$$

where $\boldsymbol{H}(\operatorname{div}, \Omega)=\left\{\boldsymbol{w} \in\left(L^{2}(\Omega)\right)^{2}: \operatorname{div} \boldsymbol{w} \in L^{2}(\Omega)\right\}$.

Now, we choose a quasi-uniform triangulation mesh $\mathscr{T}_{h}=\left\{K_{B}\right\}$ of the region $\Omega$, where $K_{B}$ represents a triangular element with $B$ as the centre of gravity, referring to Figure 1 . Let $h_{K_{B}}$ be the diameter of triangle $K_{B}$, and $h=\max \left\{h_{K_{B}}\right\}$. The nodes are defined as the midpoint of the three sides of the triangle. $P_{1}, P_{2}, \cdots, P_{M_{0}}$ represent the inner nodes and $P_{M_{0}+1}, \cdots, P_{M_{S}}$ represent the boundary nodes.

We select the $R T_{0}$ space $\boldsymbol{H}_{h}$ as the trial function space for variable $\sigma$, where

$$
\boldsymbol{H}_{h}=\left\{\boldsymbol{w}_{h} \in \boldsymbol{H}(\operatorname{div}, \Omega):\left.\boldsymbol{w}_{h}\right|_{K}=\left(a+b x_{1}, c+b x_{2}\right), \forall K \in \mathscr{T}_{h}\right\},
$$

and select $L_{h}$ as the trial function space for variable $u$, where

$$
L_{h}=\left\{v_{h} \in L^{2}(\Omega):\left.v_{h}\right|_{K} \in P_{0}(K), \forall K \in \mathscr{T}_{h}\right\} .
$$

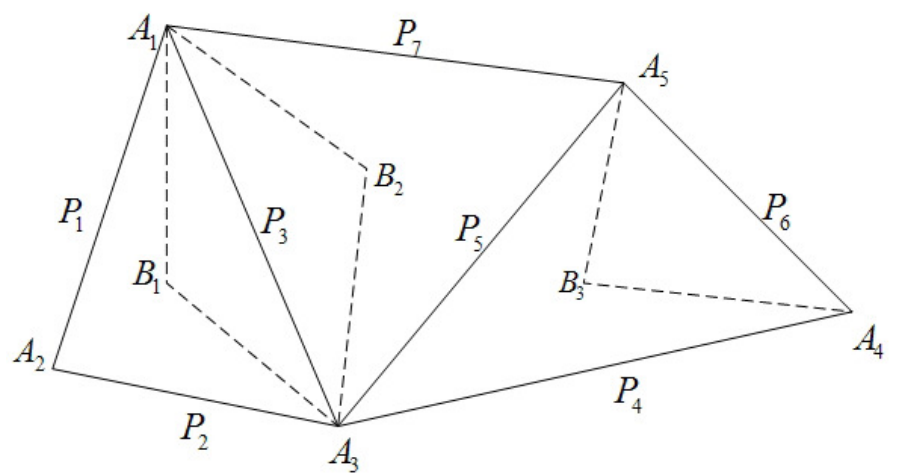

Figure 1. Primal and dual partitions.

Next, we construct the dual partition $\mathscr{T}_{h}^{*}$, which is a union of quadrilaterals and triangles. For the interior node such as $P_{3}$, referring to Figure 1 , the dual element $K_{P_{3}}^{*}$ is the quadrilateral $A_{1} B_{1} A_{3} B_{2}$, and contains triangles $K_{L}$ (with $\Delta A_{1} B_{1} A_{3}$ ) and $K_{R}$ (with $\Delta A_{1} A_{3} B_{2}$ ). For the boundary node such as $P_{6}$, the dual element $K_{P_{6}}^{*}$ is the triangle $K_{I}$ (with $\Delta A_{4} A_{5} B_{3}$ ).

Integrate (3) on the primal and dual partitions to obtain

$$
\left\{\begin{array}{l}
\text { (a) } \int_{K_{P}^{*} \cap K_{j}}(\sigma+\nabla u) \mathrm{d} X=0, j=L, R, \text { or } I, \\
\text { (b) } \int_{K_{B}}\left(\frac{\partial^{\alpha} u}{\partial t^{\alpha}}+\varepsilon \operatorname{div} \sigma+p u\right) \mathrm{d} X=\int_{K_{B}} f \mathrm{~d} X
\end{array}\right.
$$

Similar to Reference [37], the transfer operator $\gamma_{h}: H_{h} \rightarrow\left(L^{2}(\Omega)\right)^{2}$ is defined by

$$
\gamma_{h} \boldsymbol{w}_{h}=\sum_{j=1}^{M_{0}}\left(\left.\boldsymbol{w}_{h}\right|_{K_{L}}\left(P_{j}\right) \chi_{K_{j}^{*} \cap K_{L}}+\left.w_{h}\right|_{K_{R}}\left(P_{j}\right) \chi_{K_{j}^{*} \cap K_{R}}\right)+\left.\sum_{j=M_{0}+1}^{M_{S}} \boldsymbol{w}_{h}\right|_{K_{I}}\left(P_{j}\right) \chi_{K_{j}^{*}},
$$


where $\chi_{K}$ represents the characteristic function of a set $K$. We select the range $\mathbf{Y}_{h}$ of the transfer operator $\gamma_{h}$ as the test function space. Then, we apply the transfer operator $\gamma_{h}$ and rewrite (7) to obtain the following form

$$
\left\{\begin{array}{l}
(a)\left(\sigma+\nabla u, \gamma_{h} \boldsymbol{w}_{h}\right)=0, \forall \boldsymbol{w}_{h} \in \boldsymbol{H}_{h}, \\
\text { (b) }\left(\frac{\partial^{\alpha} u}{\partial t^{\alpha}}+\varepsilon \operatorname{div} \sigma+p u, v_{h}\right)=\left(f, v_{h}\right), \forall v_{h} \in L_{h} .
\end{array}\right.
$$

Making use of the Green theorem, we have

$$
\begin{aligned}
\left(\nabla u, \gamma_{h} \boldsymbol{w}_{h}\right)= & \sum_{j=1}^{M_{0}}\left(\left.\boldsymbol{w}_{h}\right|_{K_{L}}\left(P_{j}\right) \int_{\partial K_{P_{j}}^{*} \cap K_{L}} u n \mathrm{~d} s+\left.\boldsymbol{w}_{h}\right|_{K_{R}}\left(P_{j}\right) \int_{\partial K_{P_{j}}^{*} \cap K_{R}} u n \mathrm{~d} s\right) \\
& +\left.\sum_{j=M_{0}+1}^{M_{S}} \boldsymbol{w}_{h}\right|_{K_{I}}\left(P_{j}\right) \int_{\partial K_{P_{j}}^{*} \backslash \partial \Omega} u n \mathrm{~d} s \\
\doteq & b\left(\gamma_{h} \boldsymbol{w}_{h}, u\right), \forall \boldsymbol{w}_{h} \in \boldsymbol{H}_{h},
\end{aligned}
$$

where $\boldsymbol{n}$ represents the unit out-normal direction. We can easily obtain that $b\left(\gamma_{h} \boldsymbol{w}_{h}, v_{h}\right)=$ $-\left(\operatorname{div} \boldsymbol{w}_{h}, v_{h}\right), \forall \boldsymbol{w}_{h} \in \boldsymbol{H}_{h}, \forall v_{h} \in L_{h}$. Then, we rewrite (9) to the following form

$$
\left\{\begin{array}{l}
(a)\left(\sigma_{h}, \gamma_{h} \boldsymbol{w}_{h}\right)-\left(\operatorname{div} \boldsymbol{w}_{h}, u_{h}\right)=0, \forall \boldsymbol{w}_{h} \in \boldsymbol{H}_{h}, \\
(b)\left(\frac{\partial^{\alpha} u_{h}}{\partial t^{\alpha}}, v_{h}\right)+\varepsilon\left(\operatorname{div} \sigma_{h}, v_{h}\right)+\left(p u_{h}, v_{h}\right)=\left(f, v_{h}\right), \forall v_{h} \in L_{h} .
\end{array}\right.
$$

Now, let $0=t_{0}<t_{1}<\cdots<t_{N}=T$ be a equidistant grid of the time interval $[0, T]$ with step length $\tau=T / N$, for a positive integer $N$, and denote $t_{n}=n \tau, n=0,1, \cdots, N$. For a given function $\varphi$ on $[0, T]$, we denote $\varphi^{n}=\varphi\left(t_{n}\right), \partial_{t} \varphi^{n}=\frac{\varphi^{n}-\varphi^{n-1}}{\tau}$. Following References [52,53], we can approximate the time-fractional derivative $\frac{\partial^{\alpha} u(X, t)}{\partial t^{\alpha}}$ at $t=t_{n}$ as follows

$$
\begin{aligned}
\frac{\partial^{\alpha} u\left(X, t_{n}\right)}{\partial t^{\alpha}} & =\frac{1}{\Gamma(1-\alpha)} \int_{0}^{t_{n}} \frac{\partial u(X, s)}{\partial s} \frac{\mathrm{d} s}{\left(t_{n}-s\right)^{\alpha}} \\
& =\frac{\tau^{1-\alpha}}{\Gamma(2-\alpha)} \sum_{k=0}^{n-1} \hat{b}_{k} \frac{u\left(X, t_{n-k}\right)-u\left(X, t_{n-k-1}\right)}{\tau}+R_{1}^{n}(x)+R_{2}^{n}(x) \\
& =\frac{\tau^{1-\alpha}}{\Gamma(2-\alpha)} \sum_{k=0}^{n-1} \hat{b}_{k} \partial_{t} u^{n-k}+R_{t}^{n}(x) \\
& =\frac{\tau^{-\alpha}}{\Gamma(2-\alpha)} \sum_{k=0}^{n} b_{k}^{n} u^{k}+R_{t}^{n}(x),
\end{aligned}
$$

where $\hat{b}_{k}=(k+1)^{1-\alpha}-k^{1-\alpha}, b_{k}^{n}=(n-k+1)^{1-\alpha}-2(n-k)^{1-\alpha}+(n-k-1)^{1-\alpha}, b_{n}^{n}=1, b_{0}^{n}=$ $(n-1)^{1-\alpha}-n^{1-\alpha}, R_{t}^{n}(x)=R_{1}^{n}(x)+R_{2}^{n}(x)$, and

$$
\begin{aligned}
& R_{1}^{n}(x)=\frac{1}{\Gamma(1-\alpha)} \sum_{k=1}^{n} \int_{t_{k-1}}^{t_{k}} \frac{\partial u(X, s)}{\partial s} \frac{\mathrm{d} s}{\left(t_{n}-s\right)^{\alpha}}-\frac{\tau^{1-\alpha}}{\Gamma(1-\alpha)} \sum_{k=0}^{n-1} \hat{b}_{k} \frac{\partial}{\partial t} u\left(X, t_{n-k-1 / 2}\right), \\
& R_{2}^{n}(x)=\frac{\tau^{1-\alpha}}{\Gamma(2-\alpha)} \sum_{k=0}^{n-1} \hat{b}_{k}\left(\frac{\partial}{\partial t} u\left(X, t_{n-k-1 / 2}\right)-\partial_{t} u^{n-k}\right) .
\end{aligned}
$$

Denote $D_{t}^{\alpha} \varphi^{n}=\frac{\tau^{-\alpha}}{\Gamma(2-\alpha)} \sum_{k=0}^{n} b_{k}^{n} \varphi^{k}$, then we have $\frac{\partial^{\alpha} u\left(X, t_{n}\right)}{\partial t^{\alpha}}=D_{t}^{\alpha} u^{n}+R_{t}^{n}(x)$. 
Let $U^{n}$ and $Z^{n}$ be the approximate solutions of $u$ and $\sigma$ at $t=t_{n}$, respectively. Then we get the fully-discrete MFVE scheme to find $\left\{U^{n}, Z^{n}\right\} \in L_{h} \times H_{h}, n=1,2, \cdots, N$, such that

$$
\left\{\begin{array}{l}
(a)\left(Z^{n}, \gamma_{h} \mathbf{w}_{h}\right)-\left(U^{n}, \operatorname{div} \boldsymbol{w}_{h}\right)=0, \forall \boldsymbol{w}_{h} \in \boldsymbol{H}_{h}, \\
\text { (b) }\left(D_{t}^{\alpha} U^{n}, v_{h}\right)+\varepsilon\left(\operatorname{div} \mathbf{Z}^{n}, v_{h}\right)+\left(p(X) U^{n}, v_{h}\right)=\left(f^{n}, v_{h}\right), \forall v_{h} \in L_{h},
\end{array}\right.
$$

where $\left\{U^{0}, Z^{0}\right\} \in L_{h} \times \boldsymbol{H}_{h}$ satisfies

$$
\left\{\begin{array}{l}
(a)\left(U^{0}, v_{h}\right)=\left(u_{0}, v_{h}\right), \forall v_{h} \in L_{h} \\
\text { (b) }\left(Z^{0}, \gamma_{h} \boldsymbol{w}_{h}\right)-\left(U^{0}, \operatorname{div} \boldsymbol{w}_{h}\right)=0, \forall \boldsymbol{w}_{h} \in \boldsymbol{H}_{h} .
\end{array}\right.
$$

Remark 1. (1) In practical numerical calculations, we need to apply the definition of $D_{t}^{\alpha} \varphi^{n}$ to rewrite (13)(b) into the following or other equations

$\frac{1}{\Gamma(2-\alpha)}\left(U^{n}, v_{h}\right)+\varepsilon \tau^{\alpha}\left(\operatorname{div} Z^{n}, v_{h}\right)+\tau^{\alpha}\left(p(X) U^{n}, v_{h}\right)=\tau^{\alpha}\left(f^{n}, v_{h}\right)-\frac{1}{\Gamma(2-\alpha)} \sum_{k=0}^{n-1} b_{k}^{n}\left(U^{k}, v_{h}\right), \forall v_{h} \in L_{h}$.

The above equation is also used when the existence and uniqueness of discrete solutions are proved in Section 4.

(2) Compared with the standard FE methods [17-20] and FVE methods [34,35], the MFVE methods can simultaneously compute the different physical quantities about the scalar unknown variable $u$ (such as displacement) and the vector-valued auxiliary variable $\sigma$ (such as flux).

(3) In this article, we consider the Caputo fractional reaction-diffusion equations. Actually, we can apply the conversion relationship between the Caputo derivative and the Riemann-Liouville derivative to approximate the Riemann-Liouville derivative.

\section{Some Lemmas and Notations}

From (11) and (12), we can know that the truncation errors $R_{1}^{n}(x), R_{2}^{n}(x)$ and $R_{t}^{n}(x)$ will be estimated by the following lemma.

Lemma 1 ([52] (Inequalities (3.2) and (3.3))). The truncation errors $R_{1}^{n}(x), R_{2}^{n}(x)$ and $R_{t}^{n}(x)$ defined by (12) are bounded by

$$
\begin{aligned}
& \left\|R_{1}^{n}(x)\right\| \leq C \tau^{2-\alpha},\left\|R_{2}^{n}(x)\right\| \leq C \tau^{2}, \\
& \left\|R_{t}^{n}(x)\right\| \leq C\left(\tau^{2}+\tau^{2-\alpha}\right),
\end{aligned}
$$

where $C>0$ is a generic constant which is independent of space-time grid step $h$ and $\tau$.

Lemma 2 ([52] (See the proof of Theorem 3.2)). Let $\varphi^{k} \geq 0, k=0,1, \cdots, N$, which satisfy

$$
\varphi^{n} \leq-\sum_{k=1}^{n-1} b_{k}^{n} \varphi^{k}+\zeta
$$

with $\zeta>0$, then there exists a constant $C>0$ independent of space-time grid step $h$ and $\tau$ such that

$$
\varphi^{n} \leq C \tau^{-\alpha} \zeta, n=1,2, \cdots, N .
$$

Next, we give the properties of the transfer operator $\gamma_{h}$. For $\forall \mathbf{w}_{h}=\left(w_{h}^{1}, w_{h}^{2}\right) \in \boldsymbol{H}_{h}$, the discrete seminorm and the norm are defined as follows

$$
\left|\mathbf{w}_{h}\right|_{1, h}^{2}=\sum_{K \in \mathfrak{T}_{h}}\left(\left\|\nabla w_{h}^{1}\right\|_{0, K}^{2}+\left\|\nabla w_{h}^{1}\right\|_{0, K}^{2}\right),\left\|\mathbf{w}_{h}\right\|_{1, h}^{2}=\left\|\mathbf{w}_{h}\right\|^{2}+\left|\mathbf{w}_{h}\right|_{1, h}^{2} .
$$


Lemma 3 ([37] (Lemma 2.4)). The transfer operator $\gamma_{h}$ is bounded

$$
\left\|\gamma_{h} \boldsymbol{w}_{h}\right\| \leq\left\|\boldsymbol{w}_{h}\right\|, \forall \boldsymbol{w}_{h} \in \mathbf{H}_{h}
$$

Moreover, there exists a constant $C>0$ independent of $h$ such that

$$
\begin{aligned}
& \left\|\left(I-\gamma_{h}\right) \boldsymbol{w}_{h}\right\| \leq C h\left\|\boldsymbol{w}_{h}\right\|_{1, h}, \forall \boldsymbol{w}_{h} \in \mathbf{H}_{h} \\
& \left|\left(\boldsymbol{w}_{h},\left(I-\gamma_{h}\right) \boldsymbol{z}_{h}\right)\right| \leq C h\left\|\boldsymbol{w}_{h}\right\|_{1, h}\left\|\boldsymbol{z}_{h}\right\|, \forall \boldsymbol{w}_{h}, \boldsymbol{z}_{h} \in \mathbf{H}_{h} \\
& \left|\left(\boldsymbol{w},\left(I-\gamma_{h}\right) \boldsymbol{z}_{h}\right)\right| \leq C h\|\boldsymbol{w}\|_{1}\left\|\boldsymbol{z}_{h}\right\|, \forall \boldsymbol{w} \in\left(H^{1}(\Omega)\right)^{2}, \forall \boldsymbol{z}_{h} \in \mathbf{H}_{h} .
\end{aligned}
$$

Lemma 4 ([39] (Lemmas 3.3 and 3.4)). The following symmetry relation holds

$$
\left(\gamma_{h} \boldsymbol{w}_{h}, z_{h}\right)=\left(\boldsymbol{w}_{h}, \gamma_{h} z_{h}\right), \forall \boldsymbol{w}_{h}, \boldsymbol{z}_{h} \in \mathbf{H}_{h}
$$

and there exists a constant $\mu_{0}>0$ independent of $h$ such that

$$
\left(\gamma_{h} \boldsymbol{w}_{h}, \boldsymbol{w}_{h}\right) \geq \mu_{0}\left\|\boldsymbol{w}_{h}\right\|^{2}, \forall \boldsymbol{w}_{h} \in \mathbf{H}_{h}
$$

Lemma 5. Let $\left\{\boldsymbol{\psi}^{n}\right\}_{n=0}^{\infty}$ be a function sequence on $L^{2}(\Omega)$, then we have

$$
\left(\gamma_{h} \boldsymbol{\psi}^{n}, \sum_{k=0}^{n} b_{k}^{n} \boldsymbol{\psi}^{n}\right)=\frac{1}{2}\left[\left(\boldsymbol{\psi}^{n}, \gamma_{h} \boldsymbol{\psi}^{n}\right)+\sum_{k=0}^{n-1} b_{k}^{n}\left(\boldsymbol{\psi}^{k}, \gamma_{h} \boldsymbol{\psi}^{k}\right)-\sum_{k=0}^{n-1} b_{k}^{n}\left(\boldsymbol{\psi}^{n}-\boldsymbol{\psi}^{k}, \gamma_{h}\left(\boldsymbol{\psi}^{n}-\boldsymbol{\psi}^{k}\right)\right)\right]
$$

Proof. Noting that $-\sum_{k=0}^{n-1} b_{k}^{n}=1$, and applying Lemma 4, we obtain

$$
\begin{aligned}
\left(\gamma_{h} \boldsymbol{\psi}^{n}, \sum_{k=0}^{n} b_{k}^{n} \boldsymbol{\psi}^{n}\right) & =\left(\gamma_{h} \boldsymbol{\psi}^{n}, b_{n}^{n} \boldsymbol{\psi}^{n}\right)+\left(\gamma_{h} \boldsymbol{\psi}^{n}, \sum_{k=0}^{n-1} b_{k}^{n} \boldsymbol{\psi}^{n}\right) \\
& =\frac{1}{2}\left(\gamma_{h} \boldsymbol{\psi}^{n}, \boldsymbol{\psi}^{n}\right)+\frac{1}{2} \sum_{k=0}^{n-1} b_{k}^{n}\left[-\left(\gamma_{h} \boldsymbol{\psi}^{n}, \boldsymbol{\psi}^{n}\right)+\left(\gamma_{h} \boldsymbol{\psi}^{n}, \boldsymbol{\psi}^{k}\right)+\left(\gamma_{h} \boldsymbol{\psi}^{k}, \boldsymbol{\psi}^{n}\right)\right] .
\end{aligned}
$$

Applying Lemma 4, we can have the following equation

$$
-\left(\gamma_{h} \boldsymbol{\psi}^{n}, \boldsymbol{\psi}^{n}\right)+\left(\gamma_{h} \boldsymbol{\psi}^{n}, \boldsymbol{\psi}^{k}\right)+\left(\gamma_{h} \boldsymbol{\psi}^{k}, \boldsymbol{\psi}^{n}\right)=\left(\gamma_{h} \boldsymbol{\psi}^{k}, \boldsymbol{\psi}^{k}\right)-\left(\gamma_{h}\left(\boldsymbol{\psi}^{k}-\boldsymbol{\psi}^{n}\right),\left(\boldsymbol{\psi}^{k}-\boldsymbol{\psi}^{n}\right)\right) .
$$

Substituting the above equation into (15), we obtain the desired result.

Now, we introduce the generalized MFVE projection $\left(\tilde{u}_{h}, \tilde{\sigma}_{h}\right):[0, T] \rightarrow L_{h} \times \boldsymbol{H}_{h}$, satisfies

$$
\left\{\begin{array}{l}
(a)\left(\sigma-\tilde{\sigma}_{h}, \gamma_{h} \boldsymbol{w}_{h}\right)-\left(u-\tilde{u}_{h}, \operatorname{div} \boldsymbol{w}_{h}\right)=-\left(\sigma,\left(I-\gamma_{h}\right) \boldsymbol{w}_{h}\right), \forall \boldsymbol{w}_{h} \in \boldsymbol{H}_{h}, \\
(b)\left(\operatorname{div}\left(\sigma-\tilde{\sigma}_{h}\right), v_{h}\right)=0, \forall v_{h} \in L_{h} .
\end{array}\right.
$$

According to Reference [40], the above generalized MFVE projection satisfies the following estimates.

Lemma 6 ([40] (Theorems 3.1 and 3.2)). Suppose $\left(\tilde{u}_{h}, \tilde{\sigma}_{h}\right)$ satisfies (17), then there exists a constant $C>0$ independent of $h$ and $t$ such that, for $i=0,1$

$$
\begin{aligned}
& \left\|\frac{\partial^{i} \sigma}{\partial t^{i}}-\frac{\partial^{i} \tilde{\sigma}_{h}}{\partial t^{i}}\right\| \leq C h\left\|\frac{\partial^{i} \sigma}{\partial t^{i}}\right\|_{1}, \frac{\partial^{i} \sigma}{\partial t^{i}} \in\left(H^{1}(\Omega)\right)^{2}, \\
& \left\|\operatorname{div} \frac{\partial^{i} \sigma}{\partial t^{i}}-\operatorname{div} \frac{\partial^{i} \tilde{\sigma}_{h}}{\partial t^{i}}\right\| \leq C h\left\|\frac{\partial^{i} \sigma}{\partial t^{i}}\right\|_{1}, \frac{\partial^{i} \sigma}{\partial t^{i}} \in \mathbf{H}^{1}(\operatorname{div}, \Omega), \\
& \left\|\frac{\partial^{i} u}{\partial t^{i}}-\frac{\partial^{i} \tilde{u}}{\partial t^{i}}\right\| \leq \operatorname{Ch}\left(\left\|\frac{\partial^{i} \sigma}{\partial t^{i}}\right\|_{1}+\left\|\frac{\partial^{i} u}{\partial t^{i}}\right\|_{1}\right), \frac{\partial^{i} \sigma}{\partial t^{i}} \in\left(H^{1}(\Omega)\right)^{2}, \frac{\partial^{i} u}{\partial t^{i}} \in H^{1}(\Omega),
\end{aligned}
$$


where $\mathbf{H}^{1}(\operatorname{div}, \Omega)=\left\{\boldsymbol{w} \in\left(L^{2}(\Omega)\right)^{2}: \operatorname{div} \boldsymbol{w} \in H^{1}(\Omega)\right\}$.

\section{Existence, Uniqueness and Stability Analysis for the MFVE Scheme}

We first give the detailed proof of the existence and uniqueness for the MFVE scheme (13).

Theorem 1. There exists a unique solution for the MFVE scheme (13).

Proof. Let $\left\{\boldsymbol{\phi}_{i}\right\}_{i=1}^{M_{S}}$ and $\left\{\psi_{j}\right\}_{j=1}^{M_{B}}$ be the basis functions of the space $\boldsymbol{H}_{h}$ and $L_{h}$, then $\mathbf{Z}^{n} \in \boldsymbol{H}_{h}$ and $U^{n} \in L_{h}$ are expressed as follows

$$
\mathbf{Z}^{n}=\sum_{i=1}^{M_{S}} z_{i}^{n} \boldsymbol{\phi}_{i}(x), U^{n}=\sum_{j=1}^{M_{B}} u_{j}^{n} \psi_{j}(x)
$$

Substituting the above expressions into (13), and taking $\mathbf{w}_{h}=\phi_{i}\left(i=1,2, \cdots, M_{S}\right)$ and $v_{h}=$ $\psi_{j}\left(j=1,2, \cdots, M_{B}\right)$, then (13) can be expressed as a matrix form: find $\left\{\mathbf{z}^{n}, \mathbf{u}^{n}\right\}$ such that

$$
\left[\begin{array}{cc}
A_{1} & -C \\
\varepsilon \tau^{\alpha} C^{T} & \frac{1}{\Gamma(2-\alpha)} A_{2}+\tau^{\alpha} A_{3}
\end{array}\right]\left[\begin{array}{l}
\mathbf{z}^{n} \\
\mathbf{u}^{n}
\end{array}\right]=\left[\begin{array}{c}
0 \\
\tau^{\alpha} F^{n}-\frac{1}{\Gamma(2-\alpha)} \sum_{k=0}^{n-1} b_{k}^{n} A_{2} \mathbf{u}^{k}
\end{array}\right],
$$

where

$$
\begin{array}{ll}
\mathbf{z}^{n}=\left(z_{1}^{n}, z_{2}^{n}, \cdots, z_{M_{S}}^{n}\right)^{T}, & \mathbf{u}^{n}=\left(u_{1}^{n}, u_{2}^{n}, \cdots, u_{M_{B}}^{n}\right)^{T}, \\
A_{1}=\left(\left(\boldsymbol{\phi}_{i}, \gamma_{h} \boldsymbol{\phi}_{j}\right)\right)_{i, j=1, \cdots, M_{S},} & A_{2}=\left(\left(\psi_{i}, \psi_{j}\right)\right)_{i, j=1, \cdots, M_{B},} \\
A_{3}=\left(\left(p \psi_{i}, \psi_{j}\right)\right)_{i, j=1, \cdots, M_{B}}, & C=\left(\left(\psi_{i}, \operatorname{div} \boldsymbol{\phi}_{j}\right)\right)_{i=1, \cdots, M_{B}, j=1, \cdots, M_{S}}, \\
F^{n}=\left(\left(f^{n}, \psi_{j}\right)\right)_{j=1, \cdots, M_{B}}^{T} . &
\end{array}
$$

It should be noted that $A_{1}, A_{2}$ and $A_{3}$ are symmetric positive definite matrices. Noting that

$$
\left[\begin{array}{cc}
E & 0 \\
-\varepsilon \tau^{\alpha} C^{T} A_{1}^{-1} & E
\end{array}\right]\left[\begin{array}{cc}
A_{1} & -C \\
\varepsilon \tau^{\alpha} C^{T} & \frac{1}{\Gamma(2-\alpha)} A_{2}+\tau^{\alpha} A_{3}
\end{array}\right]=\left[\begin{array}{cc}
A_{1} & -C \\
0 & G
\end{array}\right],
$$

where $E$ is the identity matrix, $G=\frac{1}{\Gamma(2-\alpha)} A_{2}+\tau^{\alpha} A_{3}+\varepsilon \tau^{\alpha} C^{T} A_{1}^{-1} C$, we have that $G$ is a symmetric positive definite matrix and the coefficient matrix of linear equations (21) is invertible. Thus, linear Equations (21) have a unique solution, that is to say the MFVE scheme (13) has a unique solution. The proof of Theorem 1 has been completed.

Next, we consider the stability for the MFVE scheme (13).

Theorem 2. Let $\left\{U^{n}, \mathbf{Z}^{n}\right\}$ be the solution of the MFVE scheme (13), then there exists a constant $C>0$ independent of $h$ and $\tau$ such that

$$
\left\|U^{n}\right\| \leq C \Gamma(2-\alpha) \sup _{[0, T]}\|f(t)\|,\left\|Z^{n}\right\| \leq C \sqrt{\Gamma(2-\alpha)} \sup _{[0, T]}\|f(t)\| .
$$

Proof. Choosing $v_{h}=U^{n}$ and $w_{h}=Z^{n}$ in (13), we can obtain

$$
\left(D_{t}^{\alpha} U^{n}, U^{n}\right)+\varepsilon\left(Z^{n}, \gamma_{h} \mathbf{Z}^{n}\right)+\left(p(X) U^{n}, U^{n}\right)=\left(f^{n}, U^{n}\right) .
$$

By virtue of the definition of $D_{t}^{\alpha} U^{n}$, we derive

$$
\left(D_{t}^{\alpha} U^{n}, U^{n}\right)=\frac{\tau^{-\alpha}}{\Gamma(2-\alpha)}\left\|U^{n}\right\|^{2}+\frac{\tau^{-\alpha}}{\Gamma(2-\alpha)} \sum_{k=0}^{n-1} b_{k}^{n}\left(U^{k}, U^{n}\right) .
$$


Substituting (24) into (23), and applying Lemma 4, we have

$$
\begin{gathered}
\left\|U^{n}\right\|^{2} \leq-\sum_{k=0}^{n-1} b_{k}^{n}\left(U^{k}, U^{n}\right)+\tau^{\alpha} \Gamma(2-\alpha)\left(f^{n}, U^{n}\right) . \\
\left\|U^{n}\right\|^{2} \leq-\sum_{k=0}^{n-1} b_{k}^{n}\left\|U^{k}\right\|\left\|U^{n}\right\|+\tau^{\alpha} \Gamma(2-\alpha)\left\|f^{n}\right\|\left\|U^{n}\right\|,
\end{gathered}
$$

The above inequality leads to the following result

$$
\left\|U^{n}\right\| \leq-\sum_{k=0}^{n-1} b_{k}^{n}\left\|U^{k}\right\|+\tau^{\alpha} \Gamma(2-\alpha)\left\|f^{n}\right\| .
$$

Applying Lemma 2, we obtain

$$
\left\|U^{n}\right\| \leq C \Gamma(2-\alpha) \sup _{[0, T]}\|f(t)\| .
$$

Now, making use of (13)(a) and (14)(b), we have

$$
\left(D_{t}^{\alpha} Z^{n}, \gamma_{h} \mathbf{w}_{h}\right)-\left(D_{t}^{\alpha} U^{n}, \operatorname{div} \boldsymbol{w}_{h}\right)=0, \forall \boldsymbol{w}_{h} \in \boldsymbol{H}_{h} .
$$

Choosing $\boldsymbol{w}_{h}=Z^{n}$ in (28) and $v_{h}=D_{t}^{\alpha} U^{n}$ in (13)(b), we have

$$
\left\|D_{t}^{\alpha} U^{n}\right\|^{2}+\varepsilon\left(D_{t}^{\alpha} Z^{n}, \gamma_{h} \mathbf{Z}^{n}\right)+\left(p(X) U^{n}, D_{t}^{\alpha} U^{n}\right)=\left(f^{n}, D_{t}^{\alpha} U^{n}\right) .
$$

Apply Lemma 5 in (29) and note that

$$
-\sum_{k=0}^{n-1} b_{k}^{n}\left(\mathbf{Z}^{n}-\mathbf{Z}^{k}, \gamma_{h}\left(\mathbf{Z}^{n}-\mathbf{Z}^{k}\right)\right) \geq 0
$$

then (29) leads to

$$
\begin{aligned}
& \left\|D_{t}^{\alpha} U^{n}\right\|^{2}+\varepsilon \frac{\tau^{-\alpha}}{2 \Gamma(2-\alpha)}\left[\left(Z^{n}, \gamma_{h} Z^{n}\right)+\sum_{k=0}^{n-1} b_{k}^{n}\left(Z^{k}, \gamma_{h} Z^{k}\right)\right] \\
& \leq\left(f^{n}, D_{t}^{\alpha} U^{n}\right)-\left(p(X) U^{n}, D_{t}^{\alpha} U^{n}\right) .
\end{aligned}
$$

Applying the Cauchy-Schwarz inequality and the Young inequality in (30), we have

$$
\begin{gathered}
\left\|D_{t}^{\alpha} U^{n}\right\|^{2}+\varepsilon \frac{\tau^{-\alpha}}{2 \Gamma(2-\alpha)}\left[\left(Z^{n}, \gamma_{h} Z^{n}\right)+\sum_{k=0}^{n-1} b_{k}^{n}\left(Z^{k}, \gamma_{h} Z^{k}\right)\right] \\
\leq C\left(\left\|f^{n}\right\|^{2}+\left\|U^{n}\right\|^{2}\right)+\frac{1}{2}\left\|D_{t}^{\alpha} U^{n}\right\|^{2}
\end{gathered}
$$

Then we have

$$
\frac{1}{2}\left\|D_{t}^{\alpha} U^{n}\right\|^{2}+\varepsilon \frac{\tau^{-\alpha}}{2 \Gamma(2-\alpha)}\left[\left(Z^{n}, \gamma_{h} Z^{n}\right)+\sum_{k=0}^{n-1} b_{k}^{n}\left(Z^{k}, \gamma_{h} \mathbf{Z}^{k}\right)\right] \leq C\left(\left\|f^{n}\right\|^{2}+\left\|U^{n}\right\|^{2}\right) .
$$


Multiply (31) by $\frac{2 \Gamma(2-\alpha)}{\varepsilon \tau^{-\alpha}}$ to obtain

$$
\left(Z^{n}, \gamma_{h} Z^{n}\right) \leq-\sum_{k=0}^{n-1} b_{k}^{n}\left(Z^{k}, \gamma_{h} Z^{k}\right)+C \frac{\tau^{\alpha} \Gamma(2-\alpha)}{\varepsilon}\left(\left\|f^{n}\right\|^{2}+\left\|U^{n}\right\|^{2}\right)
$$

Applying Lemma 2 and (27), we derive

$$
\mu_{0}\left\|\mathbf{Z}^{n}\right\|^{2} \leq\left(\mathbf{Z}^{n}, \gamma_{h} \mathbf{Z}^{n}\right) \leq C \Gamma(2-\alpha)\left(\left\|f^{n}\right\|^{2}+\left\|U^{n}\right\|^{2}\right) \leq C \Gamma(2-\alpha) \sup _{[0, T]}\|f(t)\|^{2}
$$

Thus, we conclude the desired result.

\section{Convergence Analysis for the MFVE Scheme}

This section mainly studies the problem of convergence analysis for the MFVE scheme (13).

First, let $\left\{\tilde{u}_{h}, \tilde{\sigma}_{h}\right\}$ be the generalized MFVE projection of $\{u, \sigma\}$ defined by (17), thus the errors are expressed as

$$
\begin{aligned}
& u^{n}-U^{n}=\left(u^{n}-\tilde{u_{h}}\right)+\left(\tilde{u_{h}}-U^{n}\right)=\eta^{n}+\tilde{\zeta}^{n}, \\
& \sigma^{n}-\mathbf{Z}^{n}=\left(\sigma^{n}-\tilde{\sigma_{h}}\right)+\left(\tilde{\sigma_{h}}-\mathbf{Z}^{n}\right)=\boldsymbol{\rho}^{n}+\boldsymbol{\theta}^{n} .
\end{aligned}
$$

Making use of (4), (13) and (14), and applying the generalized MFVE projection, we obtain the error equations

$$
\left\{\begin{array}{l}
(a)\left(\xi^{n}, \operatorname{div}_{h}\right)=\left(\boldsymbol{\theta}^{n}, \gamma_{h} \mathbf{w}_{h}\right), \forall \mathbf{w}_{h} \in \boldsymbol{H}_{h}, \\
(b)\left(D_{t}^{\alpha} \xi^{n}, v_{h}\right)+\varepsilon\left(\operatorname{div} \boldsymbol{\theta}^{n}, v_{h}\right)+\left(p \xi^{n}, v_{h}\right) \\
\quad=-\left(R_{t}^{n}, v_{h}\right)-\left(D_{t}^{\alpha} \eta^{n}, v_{h}\right)-\left(p \eta^{n}, v_{h}\right), \forall v_{h} \in L_{h},
\end{array}\right.
$$

and

$$
\left\{\begin{array}{l}
(a)\left(\eta^{0}+\xi^{0}, v_{h}\right)=0, \forall v_{h} \in L_{h} \\
(b)\left(\xi^{0}, \operatorname{div}_{h}\right)=\left(\boldsymbol{\theta}^{0}, \gamma_{h} \mathbf{w}_{h}\right), \forall \mathbf{w}_{h} \in \boldsymbol{H}_{h}
\end{array}\right.
$$

Theorem 3. Let $\{u, \sigma\}$ and $\left\{U^{n}, \mathbf{Z}^{n}\right\}$ be the solutions of the system (4) and (13), respectively, then there exists a constant $C>0$ independent of $h$ and $\tau$ such that

$$
\begin{aligned}
& \max _{1 \leq n \leq N}\left\|u^{n}-U^{n}\right\|+\max _{1 \leq n \leq N}\left\|\sigma^{n}-Z^{n}\right\| \leq C\left(\tau^{2-\alpha}+h\right), \\
& \max _{1 \leq n \leq N}\left\|\sigma^{n}-Z^{n}\right\|_{\mathbf{H}(\operatorname{div}, \Omega)} \leq C\left(\tau^{2-\frac{3}{2} \alpha}+h \tau^{-\frac{\alpha}{2}}+\tau^{2-\alpha}+h\right) .
\end{aligned}
$$

Proof. Choosing $v_{h}=\xi^{n}$ and $w_{h}=\boldsymbol{\theta}^{n}$ in (33), we have

$$
\left(D_{t}^{\alpha} \xi^{n}, \xi^{n}\right)+\epsilon\left(\boldsymbol{\theta}^{n}, \gamma_{h} \boldsymbol{\theta}^{n}\right)+\left(p \xi^{n}, \xi^{n}\right)=-\left(R_{t}^{n}, \xi^{n}\right)-\left(D_{t}^{\alpha} \eta^{n}, \xi^{n}\right)-\left(p \eta^{n}, \xi^{n}\right) .
$$

Noting the fact that

$$
\left(D_{t}^{\alpha} \xi^{n}, \xi^{n}\right)=\frac{\tau^{-\alpha}}{\Gamma(2-\alpha)}\left(\xi^{n}, \xi^{n}\right)+\frac{\tau^{-\alpha}}{\Gamma(2-\alpha)} \sum_{k=0}^{n-1} b_{k}^{n}\left(\xi^{k}, \xi^{n}\right)
$$


we can rewrite (35) as follows

$$
\begin{aligned}
& \left(\xi^{n}, \xi^{n}\right)+\tau^{\alpha} \Gamma(2-\alpha) \varepsilon\left(\boldsymbol{\theta}^{n}, \gamma_{h} \boldsymbol{\theta}^{n}\right)+\tau^{\alpha} \Gamma(2-\alpha)\left(p \xi^{n}, \xi^{n}\right) \\
& =-\sum_{k=0}^{n-1} b_{k}^{n}\left(\xi^{k}, \xi^{n}\right)-\tau^{\alpha} \Gamma(2-\alpha)\left(R_{t}^{n}, \xi^{n}\right) \\
& \quad-\tau^{\alpha} \Gamma(2-\alpha)\left(D_{t}^{\alpha} \eta^{n}, \xi^{n}\right)-\tau^{\alpha} \Gamma(2-\alpha)\left(p \eta^{n}, \xi^{n}\right) .
\end{aligned}
$$

Apply Lemma 4 in (37) to obtain

$$
\begin{aligned}
\left\|\xi^{n}\right\|^{2} \leq & -\sum_{k=0}^{n-1} b_{k}^{n}\left(\xi^{k}, \xi^{n}\right)-\tau^{\alpha} \Gamma(2-\alpha)\left(R_{t}^{n}, \xi^{n}\right) \\
& -\tau^{\alpha} \Gamma(2-\alpha)\left(D_{t}^{\alpha} \eta^{n}, \xi^{n}\right)-\tau^{\alpha} \Gamma(2-\alpha)\left(p \eta^{n}, \xi^{n}\right) \doteq \sum_{i=1}^{4} F_{i} .
\end{aligned}
$$

Now, we estimate the four terms $F_{i}(i=1,2,3,4)$ on the right-hand side of (38). Noting that $b_{k}^{n}<0(0 \leq k<n)$, and applying the Cauchy-Schwarz inequality, we can obtain

$$
\begin{aligned}
& \left|F_{1}\right|=-\sum_{k=0}^{n-1} b_{k}^{n}\left(\xi^{k}, \xi^{n}\right) \leq-\sum_{k=0}^{n-1} b_{k}^{n}\left\|\xi^{k}\right\|\left\|\xi^{n}\right\|, \\
& \left|F_{2}\right|=\left|\tau^{\alpha} \Gamma(2-\alpha)\left(R_{t}^{n}, \xi^{n}\right)\right| \leq \tau^{\alpha} \Gamma(2-\alpha)\left\|R_{t}^{n}\right\|\left\|\xi^{n}\right\|, \\
& \left|F_{4}\right|=\left|\tau^{\alpha} \Gamma(2-\alpha)\left(p \eta^{n}, \xi^{n}\right)\right| \leq \tau^{\alpha} \Gamma(2-\alpha)\left\|\eta^{n}\right\|\left\|\xi^{n}\right\| .
\end{aligned}
$$

Applying $D_{t}^{\alpha} \eta^{n}=\frac{\tau^{-\alpha}}{\Gamma(2-\alpha)} \sum_{k=0}^{n-1} \tilde{b_{k}} \partial_{t} \eta^{n-k}, \sum_{k=0}^{n-1} \tilde{b_{k}}=n^{1-\alpha}$ and Lemma 6, we obtain

$$
\begin{aligned}
\left\|\partial_{t} \eta^{n-k}\right\|= & \left\|\frac{1}{\tau} \int_{t_{n-k-1}}^{t_{n-k}} \eta_{t} \mathrm{~d} t\right\| \leq h\left(\left\|u_{t}\right\|_{L^{\infty}\left(H^{1}\right)}+\left\|\sigma_{t}\right\|_{L^{\infty}\left(\boldsymbol{H}^{1}\right)}\right), \\
\left\|D_{t}^{\alpha} \eta^{n}\right\| & \leq \frac{\tau^{1-\alpha}}{\Gamma(2-\alpha)} n^{1-\alpha} h\left(\left\|u_{t}\right\|_{L^{\infty}\left(H^{1}\right)}+\left\|\sigma_{t}\right\|_{L^{\infty}\left(\boldsymbol{H}^{1}\right)}\right) \\
& \leq C T^{1-\alpha} h\left(\left\|u_{t}\right\|_{L^{\infty}\left(H^{1}\right)}+\left\|\sigma_{t}\right\|_{L^{\infty}\left(\boldsymbol{H}^{1}\right)}\right) .
\end{aligned}
$$

Thus, the $F_{3}$ can be estimated by the following result

$$
\begin{aligned}
& \left|F_{3}\right|=\left|\tau^{\alpha} \Gamma(2-\alpha)\left(D_{t}^{\alpha} \eta^{n}, \xi^{n}\right)\right| \leq \tau^{\alpha} \Gamma(2-\alpha)\left\|D_{t}^{\alpha} \eta^{n}\right\|\left\|\xi^{n}\right\| \\
& \quad \leq C \tau^{\alpha} h\left(\left\|u_{t}\right\|_{L^{\infty}\left(H^{1}\right)}+\left\|\sigma_{t}\right\|_{L^{\infty}\left(\boldsymbol{H}^{1}\right)}\right)\left\|\xi^{n}\right\| .
\end{aligned}
$$

Then, combining the estimates of $F_{i}(i=1,2,3,4)$, we have

$$
\begin{aligned}
\left\|\xi^{n}\right\| \leq & -\sum_{k=0}^{n-1} b_{k}^{n}\left\|\xi^{k}\right\|+\tau^{\alpha} \Gamma(2-\alpha)\left\|R_{t}^{n}\right\|+\tau^{\alpha} \Gamma(2-\alpha)\left\|\eta^{n}\right\| \\
& +C \tau^{\alpha} h\left(\left\|u_{t}\right\|_{L^{\infty}\left(H^{1}\right)}+\left\|\sigma_{t}\right\|_{L^{\infty}\left(H^{1}\right)}\right) .
\end{aligned}
$$

Applying Lemma 2, we obtain

$$
\left\|\xi^{n}\right\| \leq C \Gamma(2-\alpha)\left\|R_{t}^{n}\right\|+C \Gamma(2-\alpha)\left\|\eta^{n}\right\|+C h\left(\left\|u_{t}\right\|_{L^{\infty}\left(H^{1}\right)}+\left\|\sigma_{t}\right\|_{L^{\infty}\left(H^{1}\right)}\right) .
$$

To estimate $\left\|\sigma^{n}-\mathbf{Z}^{n}\right\|$, we apply (33)(a) and (34)(b) to obtain

$$
\left(D_{t}^{\alpha} \xi^{n}, \operatorname{div}_{h}\right)=\left(D_{t}^{\alpha} \boldsymbol{\theta}^{n}, \gamma_{h} \mathbf{w}_{h}\right), \forall \mathbf{w}_{h} \in \boldsymbol{H}_{h} .
$$


Choose $v_{h}=D_{t}^{\alpha} \xi^{n}$ in (33)(b) and $\mathbf{w}_{h}=\boldsymbol{\theta}^{n}$ in (47) to obtain

$$
\begin{aligned}
& \left(D_{t}^{\alpha} \xi^{n}, D_{t}^{\alpha} \xi^{n}\right)+\varepsilon\left(D_{t}^{\alpha} \boldsymbol{\theta}^{n}, \gamma_{h} \boldsymbol{\theta}^{n}\right) \\
& \quad=-\left(R_{t}^{n}, D_{t}^{\alpha} \xi^{n}\right)-\left(D_{t}^{\alpha} \eta^{n}, D_{t}^{\alpha} \xi^{n}\right)-\left(p \xi^{n}, D_{t}^{\alpha} \xi^{n}\right)-\left(p \eta^{n}, D_{t}^{\alpha} \xi^{n}\right) .
\end{aligned}
$$

Applying the Cauchy-Schwarz inequality and the Young inequality in (48), we can get

$$
\left\|D_{t}^{\alpha} \xi^{n}\right\|^{2}+\varepsilon\left(D_{t}^{\alpha} \boldsymbol{\theta}^{n}, \gamma_{h} \boldsymbol{\theta}^{n}\right) \leq C\left(\left\|R_{t}^{n}\right\|^{2}+\left\|D_{t}^{\alpha} \eta^{n}\right\|^{2}+\left\|\xi^{n}\right\|^{2}+\left\|\eta^{n}\right\|^{2}\right)+\frac{1}{2}\left\|D_{t}^{\alpha} \xi^{n}\right\|^{2} .
$$

With the help of Lemma 5 in (49), we have

$$
\begin{aligned}
& \frac{1}{2}\left\|D_{t}^{\alpha} \xi^{n}\right\|^{2}+\frac{\varepsilon \tau^{-\alpha}}{2 \Gamma(2-\alpha)}\left[\left(\boldsymbol{\theta}^{n}, \gamma_{h} \boldsymbol{\theta}^{n}\right)+\sum_{k=0}^{n-1} b_{k}^{n}\left(\boldsymbol{\theta}^{k}, \gamma_{h} \boldsymbol{\theta}^{k}\right)-\sum_{k=0}^{n-1} b_{k}^{n}\left(\boldsymbol{\theta}^{n}-\boldsymbol{\theta}^{k}, \gamma_{h}\left(\boldsymbol{\theta}^{n}-\boldsymbol{\theta}^{k}\right)\right)\right] \\
& \quad \leq C\left(\left\|R_{t}^{n}\right\|^{2}+\left\|D_{t}^{\alpha} \eta^{n}\right\|^{2}+\left\|\xi^{n}\right\|^{2}+\left\|\eta^{n}\right\|^{2}\right) .
\end{aligned}
$$

Multiply the above inequality by $\frac{2}{\varepsilon} \tau^{\alpha} \Gamma(2-\alpha)$ to get

$$
\left(\boldsymbol{\theta}^{n}, \gamma_{h} \boldsymbol{\theta}^{n}\right) \leq-\sum_{k=0}^{n-1} b_{k}^{n}\left(\boldsymbol{\theta}^{k}, \gamma_{h} \boldsymbol{\theta}^{k}\right)+\frac{C \tau^{\alpha} \Gamma(2-\alpha)}{\varepsilon}\left(\left\|R_{t}^{n}\right\|^{2}+\left\|D_{t}^{\alpha} \eta^{n}\right\|^{2}+\left\|\xi^{n}\right\|^{2}+\left\|\eta^{n}\right\|^{2}\right) .
$$

Applying Lemma 2 and Lemma 4, we obtain

$$
\mu_{0}\left\|\boldsymbol{\theta}^{n}\right\|^{2} \leq\left(\boldsymbol{\theta}^{n}, \gamma_{h} \boldsymbol{\theta}^{n}\right) \leq C\left(\left\|R_{t}^{n}\right\|^{2}+\left\|D_{t}^{\alpha} \eta^{n}\right\|^{2}+\left\|\xi^{n}\right\|^{2}+\left\|\eta^{n}\right\|^{2}\right) .
$$

Next, we estimate $\left\|\sigma^{n}-\mathbf{Z}^{n}\right\|_{\boldsymbol{H}(\operatorname{div}, \Omega)}$. Choose $v_{h}=\operatorname{div} \boldsymbol{\theta}^{n}$ in (33)(b) and $\mathbf{w}_{h}=\boldsymbol{\theta}^{n}$ in (47) to obtain

$$
\begin{aligned}
& \left(D_{t}^{\alpha} \boldsymbol{\theta}^{n}, \gamma_{h} \boldsymbol{\theta}^{n}\right)+\varepsilon\left\|\operatorname{div} \boldsymbol{\theta}^{n}\right\|^{2} \\
& \quad=-\left(R_{t}^{n}, \operatorname{div} \boldsymbol{\theta}^{n}\right)-\left(D_{t}^{\alpha} \eta^{n}, \operatorname{div} \boldsymbol{\theta}^{n}\right)-\left(r \xi^{n}, \operatorname{div} \boldsymbol{\theta}^{n}\right)-\left(r \eta^{n}, \operatorname{div} \boldsymbol{\theta}^{n}\right) .
\end{aligned}
$$

Then, (53) leads to the following result Apply the Cauchy-Schwarz inequality and the Young inequality in (53) to obtain

$$
\frac{\varepsilon}{2}\left\|\operatorname{div} \boldsymbol{\theta}^{n}\right\|^{2} \leq-\left(D_{t}^{\alpha} \boldsymbol{\theta}^{n}, \gamma_{h} \boldsymbol{\theta}^{n}\right)+C\left(\left\|R_{t}^{n}\right\|^{2}+\left\|D_{t}^{\alpha} \eta^{n}\right\|^{2}+\left\|\xi^{n}\right\|^{2}+\left\|\eta^{n}\right\|^{2}\right) .
$$

Noting that $\left(\boldsymbol{\theta}^{n}, \gamma_{h} \boldsymbol{\theta}^{n}\right) \geq 0$ and $b_{k}^{n}<0(0 \leq k<n)$, we have

$$
\begin{aligned}
-\left(D_{t}^{\alpha} \boldsymbol{\theta}^{n}, \gamma_{h} \boldsymbol{\theta}^{n}\right) & =\frac{\tau^{-\alpha}}{\Gamma(2-\alpha)}\left[\sum_{k=0}^{n-1}\left(-b_{k}^{n}\right)\left(\boldsymbol{\theta}^{k}, \gamma_{h} \boldsymbol{\theta}^{n}\right)-\left(\boldsymbol{\theta}^{n}, \gamma_{h} \boldsymbol{\theta}^{n}\right)\right] \\
& \leq \frac{\tau^{-\alpha}}{\Gamma(2-\alpha)} \sum_{k=0}^{n-1}\left(-b_{k}^{n}\right)\left\|\boldsymbol{\theta}^{k}\right\|\left\|\boldsymbol{\theta}^{n}\right\| .
\end{aligned}
$$

Making use of $\sum_{k=0}^{n-1}\left(-b_{k}^{n}\right)=1$, we have

$$
-\left(D_{t}^{\alpha} \boldsymbol{\theta}^{n}, \gamma_{h} \boldsymbol{\theta}^{n}\right) \leq C \frac{\tau^{-\alpha}}{\Gamma(2-\alpha)}\left(\tau^{2-\alpha}+h\right)^{2} .
$$

Replace $-\left(D_{t}^{\alpha} \boldsymbol{\theta}^{n}, \gamma_{h} \boldsymbol{\theta}^{n}\right)$ with the above result in (54) to obtain

$$
\frac{\varepsilon}{2}\left\|\operatorname{div} \boldsymbol{\theta}^{n}\right\|^{2} \leq C \tau^{-\alpha}\left(\tau^{2-\alpha}+h\right)^{2} .
$$

Finally, apply Lemma 1 and Lemma 6 with (46), (52) and (57) to complete the proof. 


\section{Numerical Examples}

For examining the feasibility and effectiveness of the MFVE scheme, we consider two numerical examples with one-dimensional and two-dimensional spatial regions.

Example 1. We consider the following time-fractional reaction-diffusion equation in one-dimensional spatial regions

$$
\begin{cases}\frac{\partial^{\alpha} u(x, t)}{\partial t^{\alpha}}-\varepsilon u_{x x}(x, t)+p(x) u(x, t)=f(x, t), & (x, t) \in \Omega \times J, \\ u(a, t)=u(b, t)=0, & t \in \bar{J}, \\ u(x, 0)=u_{0}(x), & x \in \bar{\Omega} .\end{cases}
$$

where $\Omega=(a, b)$ and $J=(0, T]$. We also introduce an auxiliary variable $\sigma(x, t)=-u_{x}(x, t)$, and rewrite the Equation (58) as the first-order coupled system. As in Reference [41], we establish the primal partition $T_{h}$ and dual partition $T_{h}^{*}$, and choose the space $H_{h}^{*} \times L_{h}^{*}$ as the trial function space, where

$$
\begin{gathered}
H_{h}^{*}=\left\{w_{h} \in H^{1}(\Omega): w_{h} \in P_{1}(A), \forall A \in T_{h}\right\}, \\
L_{h}^{*}=\left\{v_{h} \in L^{2}(\Omega):\left.w_{h}\right|_{A} \in P_{0}(A), \forall A \in T_{h}\right\} .
\end{gathered}
$$

As in Reference [41], we also define transfer operator $\gamma_{h}^{*}: H_{h} \rightarrow L^{2}(\Omega)$ by

$$
\gamma_{h}^{*} w_{h}=\sum_{i=0}^{M_{T}} w_{h}\left(x_{i}\right) \chi_{A_{i}^{*}}, \forall w_{h} \in H_{h}^{*}
$$

where $A_{i}^{*} \in T_{h}^{*}$ and $M_{T}$ is the number of spatial nodes.

The transfer operator $\gamma_{h}^{*}$ also satisfy properties similar to Lemmas 3-5 (see Lemmas 2.1-2.4 in Reference [41] for details). By applying operator $\gamma_{h}^{*}$, we can construct the MFVE scheme and obtain the existence, uniqueness, stability and convergence results, which are very similar to Theorems 1-3, and so we do not repeat the content and process.

Now we choose $(a, b)=(0,1), T=2, \varepsilon=1, p(x)=1+x^{2}$, the initial function $u_{0}(x)=$ $\sin (2 \pi x)$ and the source function $f(x, t)=\left(\frac{2}{\Gamma(3-\alpha)} t^{2-\alpha}+4 \pi^{2}\left(1+t^{2}\right)+\left(1+x^{2}\right)\left(1+t^{2}\right)\right) \sin (2 \pi x)$ in (58). Then we can get the exact solution $u(x, t)=\left(1+t^{2}\right) \sin (2 \pi x)$, and auxiliary variable $\sigma(x, t)=$ $-2 \pi\left(1+t^{2}\right) \cos (2 \pi x)$.

The numerical simulation results with parameter $\alpha=0.1,0.3,0.5,0.7,0.9$ are given in Tables $1-6$. We fix the spatial step length $h=1 / 10,000$, select the time step length $\tau=1 / 10,1 / 20,1 / 40,1 / 80$, and give the error results of $u$ (in $L^{\infty}\left(L^{2}(\Omega)\right)$-norm) and $\sigma$ (in $L^{\infty}\left(L^{2}(\Omega)\right)$-norm and $L^{\infty}\left(H^{1}(\Omega)\right.$ )-norm) in Tables $1-5$. It is easy to see that the orders of time convergence are approximate to $2-\alpha$ which are consistent with the theoretical results in Theorem 3. Moreover, taking different parameter $\alpha$, fixing the time step length $\tau=1 / 1000$, and selecting the spatial step length $h=1 / 10,1 / 20,1 / 40,1 / 80$, we also do some numerical experiments to examine the orders of spatial convergence. The numerical results show that the orders of spatial convergence are approximate to 2 which are larger than the theoretical results. The numerical simulation results with parameter $\alpha=0.1$ are given in Table 6 , and the other results with different parameter $\alpha$ are similar, and we will not repeat the description. 
Table 1. Error results with $\alpha=0.1$ and $h=1 \times 10^{-4}$ for Example 1 .

\begin{tabular}{ccccccc}
\hline$\tau$ & $\|\boldsymbol{u}-\boldsymbol{U}\|_{L^{\infty}\left(L^{2}\right)}$ & Order & $\|\sigma-Z\|_{L^{\infty}\left(L^{2}\right)}$ & Order & $\|\sigma-Z\|_{L^{\infty}\left(H^{1}\right)}$ & Order \\
\hline $1 / 10$ & $2.0141 \times 10^{-5}$ & & $1.2727 \times 10^{-4}$ & & $8.0744 \times 10^{-4}$ & \\
$1 / 20$ & $5.7570 \times 10^{-6}$ & 1.8067 & $3.6900 \times 10^{-5}$ & 1.7862 & $2.3250 \times 10^{-4}$ & 1.7961 \\
$1 / 40$ & $1.5713 \times 10^{-6}$ & 1.8734 & $1.0598 \times 10^{-5}$ & 1.7998 & $6.5160 \times 10^{-5}$ & 1.8352 \\
$1 / 80$ & $4.0524 \times 10^{-7}$ & 1.9551 & $3.0282 \times 10^{-6}$ & 1.8073 & $1.7300 \times 10^{-5}$ & 1.9132 \\
\hline
\end{tabular}

Table 2. Error results with $\alpha=0.3$ and $h=1 \times 10^{-4}$ for Example 1 .

\begin{tabular}{ccccccc}
\hline$\tau$ & $\|\boldsymbol{u}-\boldsymbol{U}\|_{L^{\infty}\left(\boldsymbol{L}^{2}\right)}$ & Order & $\|\sigma-Z\|_{L^{\infty}\left(L^{2}\right)}$ & Order & $\|\sigma-Z\|_{L^{\infty}\left(H^{1}\right)}$ & Order \\
\hline $1 / 10$ & $9.1658 \times 10^{-5}$ & & $5.7659 \times 10^{-4}$ & & $3.6661 \times 10^{-3}$ & \\
$1 / 20$ & $2.9166 \times 10^{-5}$ & 1.6520 & $1.8397 \times 10^{-4}$ & 1.6481 & $1.1682 \times 10^{-3}$ & 1.6500 \\
$1 / 40$ & $9.1566 \times 10^{-6}$ & 1.6714 & $5.8259 \times 10^{-5}$ & 1.6589 & $3.6839 \times 10^{-4}$ & 1.6650 \\
$1 / 80$ & $2.8149 \times 10^{-6}$ & 1.7017 & $1.8350 \times 10^{-5}$ & 1.6667 & $1.1448 \times 10^{-4}$ & 1.6861 \\
\hline
\end{tabular}

Table 3. Error results with $\alpha=0.5$ and $h=1 \times 10^{-4}$ for Example 1 .

\begin{tabular}{ccccccc}
\hline$\tau$ & $\|\boldsymbol{u}-\boldsymbol{U}\|_{L^{\infty}\left(L^{2}\right)}$ & Order & $\|\sigma-Z\|_{L^{\infty}\left(L^{2}\right)}$ & Order & $\|\sigma-Z\|_{L^{\infty}\left(H^{1}\right)}$ & Order \\
\hline $1 / 10$ & $2.4301 \times 10^{-4}$ & & $1.5275 \times 10^{-3}$ & & $9.7159 \times 10^{-3}$ & \\
$1 / 20$ & $8.7040 \times 10^{-5}$ & 1.4813 & $5.4757 \times 10^{-4}$ & 1.4800 & $3.4815 \times 10^{-3}$ & 1.4806 \\
$1 / 40$ & $3.0999 \times 10^{-5}$ & 1.4895 & $1.9549 \times 10^{-4}$ & 1.4860 & $1.2414 \times 10^{-3}$ & 1.4877 \\
$1 / 80$ & $1.0963 \times 10^{-5}$ & 1.4996 & $6.9596 \times 10^{-5}$ & 1.4900 & $4.4052 \times 10^{-4}$ & 1.4948 \\
\hline
\end{tabular}

Table 4. Error results with $\alpha=0.7$ and $h=1 \times 10^{-4}$ for Example 1 .

\begin{tabular}{ccccccc}
\hline$\tau$ & $\|\boldsymbol{u}-\boldsymbol{U}\|_{L^{\infty}\left(L^{2}\right)}$ & Order & $\|\sigma-Z\|_{L^{\infty}\left(L^{2}\right)}$ & Order & $\|\sigma-Z\|_{L^{\infty}\left(H^{1}\right)}$ & Order \\
\hline $1 / 10$ & $5.5980 \times 10^{-4}$ & & $3.5177 \times 10^{-3}$ & & $2.2378 \times 10^{-2}$ & \\
$1 / 20$ & $2.2823 \times 10^{-4}$ & 1.2944 & $1.4346 \times 10^{-3}$ & 1.2940 & $9.1248 \times 10^{-3}$ & 1.2942 \\
$1 / 40$ & $9.2857 \times 10^{-5}$ & 1.2974 & $5.8412 \times 10^{-4}$ & 1.2963 & $3.7140 \times 10^{-3}$ & 1.2968 \\
$1 / 80$ & $3.7703 \times 10^{-5}$ & 1.3003 & $2.3761 \times 10^{-4}$ & 1.2977 & $1.5094 \times 10^{-3}$ & 1.2990 \\
\hline
\end{tabular}

Table 5. Error results with $\alpha=0.9$ and $h=1 \times 10^{-4}$ for Example 1 .

\begin{tabular}{ccccccc}
\hline$\tau$ & $\|\boldsymbol{u}-\boldsymbol{U}\|_{L^{\infty}\left(\boldsymbol{L}^{2}\right)}$ & Order & $\|\sigma-Z\|_{L^{\infty}\left(L^{2}\right)}$ & Order & $\|\sigma-Z\|_{L^{\infty}\left(H^{1}\right)}$ & Order \\
\hline $1 / 10$ & $1.2043 \times 10^{-3}$ & & $7.5667 \times 10^{-3}$ & & $4.8138 \times 10^{-2}$ & \\
$1 / 20$ & $5.6212 \times 10^{-4}$ & 1.0992 & $3.5323 \times 10^{-3}$ & 1.0991 & $2.2471 \times 10^{-2}$ & 1.0991 \\
$1 / 40$ & $2.6227 \times 10^{-4}$ & 1.0998 & $1.6485 \times 10^{-3}$ & 1.0995 & $1.0486 \times 10^{-2}$ & 1.0997 \\
$1 / 80$ & $1.2232 \times 10^{-4}$ & 1.1004 & $7.6919 \times 10^{-4}$ & 1.0997 & $4.8914 \times 10^{-3}$ & 1.1001 \\
\hline
\end{tabular}

Table 6. Error results with $\alpha=0.1$ and $\tau=1 \times 10^{-3}$ for Example 1 .

\begin{tabular}{ccccccc}
\hline $\boldsymbol{h}$ & $\|\boldsymbol{u}-\boldsymbol{U}\|_{L^{\infty}\left(L^{2}\right)}$ & Order & $\|\sigma-Z\|_{L^{\infty}\left(L^{2}\right)}$ & Order & $\|\sigma-Z\|_{L^{\infty}\left(H^{1}\right)}$ & Order \\
\hline $1 / 10$ & $1.0969 \times 10^{-1}$ & & $2.0021 \times 10^{-2}$ & & 2.1604 & \\
$1 / 20$ & $2.8077 \times 10^{-2}$ & 1.9660 & $5.0148 \times 10^{-3}$ & 1.9973 & $5.4230 \times 10^{-1}$ & 1.9942 \\
$1 / 40$ & $7.0602 \times 10^{-3}$ & 1.9916 & $1.2593 \times 10^{-3}$ & 1.9936 & $1.3571 \times 10^{-1}$ & 1.9985 \\
$1 / 80$ & $1.7676 \times 10^{-3}$ & 1.9979 & $3.1584 \times 10^{-4}$ & 1.9953 & $3.3936 \times 10^{-2}$ & 1.9997 \\
\hline
\end{tabular}

Example 2. We consider the following time-fractional reaction-diffusion equation in two-dimensional spatial regions

$$
\begin{cases}\frac{\partial^{\alpha} u(X, t)}{\partial t^{\alpha}}-\varepsilon \Delta u(X, t)+p(X) u(X, t)=f(X, t), & (X, t) \in \Omega \times J, \\ u(X, t)=0, & (X, t) \in \partial \Omega \times \bar{J}, \\ u(X, 0)=\sin \left(2 \pi x_{1}\right) \sin \left(2 \pi x_{2}\right), & X=\left(x_{1}, x_{2}\right) \in \bar{\Omega},\end{cases}
$$


where $\Omega=(0,1) \times(0,1), J=(0,1], \varepsilon=1, p(X)=1+x_{1}^{2}+x_{2}^{2}$, and the source function $f(X, t)=\left(\frac{2}{\Gamma(3-\alpha)} t^{2-\alpha}+8 \pi^{2}\left(1+t^{2}\right)+\left(1+x_{1}^{2}+x_{2}^{2}\right)\left(1+t^{2}\right)\right) \sin \left(2 \pi x_{1}\right) \sin \left(2 \pi x_{2}\right)$. Then, we can obtain the exact solutions

$$
\begin{aligned}
& u(X, t)=\left(1+t^{2}\right) \sin \left(2 \pi x_{1}\right) \sin \left(2 \pi x_{2}\right), \\
& \sigma(X, t)=\left(-2 \pi\left(1+t^{2}\right) \cos \left(2 \pi x_{1}\right) \sin \left(2 \pi x_{2}\right),-2 \pi\left(1+t^{2}\right) \sin \left(2 \pi x_{1}\right) \cos \left(2 \pi x_{2}\right)\right) .
\end{aligned}
$$

In this example, we do some numerical experiments with different parameter $\alpha$ and mesh size $h=\sqrt{2} \tau$, and apply the fifth-order Gauss integral formula in triangular regions to calculate the errors of $u$ (in $L^{\infty}\left(L^{2}(\Omega)\right)$-norm) and $\sigma$ (in $L^{\infty}\left(\mathbf{L}^{2}(\Omega)\right.$-norm and $L^{\infty}(\boldsymbol{H}(\mathrm{div}, \Omega))$-norm, where $\left.\mathbf{L}^{2}(\Omega)=\left(L^{2}(\Omega)\right)^{2}\right)$. The numerical results show that the orders of convergence are approximate to 1 . The numerical simulation results are given in Table 7 with parameter $\alpha=0.1$ and mesh sizes $h=\sqrt{2} \tau=\frac{\sqrt{2}}{10}, \frac{\sqrt{2}}{20}, \frac{\sqrt{2}}{40}, \frac{\sqrt{2}}{80}$, and the other results with different parameter $\alpha$ are similar. The graphs of numerical solutions for variables $u$ and $\sigma$ at time parameter $t=1$ with a space mesh size $h=\sqrt{2} \tau=\frac{\sqrt{2}}{40}$ are given in Figures 2 and 3, respectively. Because the trial function spaces $L_{h}$ (defined in (6)) and $\boldsymbol{H}_{h}$ (defined in (5)) are not continuous function spaces, the surfaces in Figures 2 and 3 agree with the properties of the trial function spaces. The figures and numerical results show that the proposed MFVE method for the time-fractional reaction-diffusion equations in two-dimensional spatial regions is feasible and effective.

Table 7. Error results with $\alpha=0.1$ and $h=\sqrt{2} \tau$ for Example 2 .

\begin{tabular}{ccccccc}
\hline $\boldsymbol{\tau}, \boldsymbol{h}$ & $\|\boldsymbol{u}-\boldsymbol{U}\|_{L^{\infty}\left(\mathbf{L}^{2}\right)}$ & Order & $\|\sigma-\mathbf{Z}\|_{L^{\infty}\left(\mathbf{L}^{2}\right)}$ & Order & $\|\sigma-\mathbf{Z}\|_{L^{\infty}(\boldsymbol{H}(\mathrm{div}))}$ & Order \\
\hline $1 / 10, \sqrt{2} / 10$ & $2.5821 \times 10^{-1}$ & & 2.0257 & & $2.0380 \times 10^{1}$ & \\
$1 / 20, \sqrt{2} / 20$ & $1.3045 \times 10^{-1}$ & 0.9850 & 1.0089 & 1.0057 & $1.0335 \times 10^{1}$ & 0.9796 \\
$1 / 40, \sqrt{2} / 40$ & $6.5394 \times 10^{-2}$ & 0.9963 & $5.0386 \times 10^{-1}$ & 1.0017 & 5.1860 & 0.9949 \\
$1 / 80, \sqrt{2} / 80$ & $3.2718 \times 10^{-2}$ & 0.9991 & $2.5185 \times 10^{-1}$ & 1.0004 & 2.5953 & 0.9987 \\
\hline
\end{tabular}

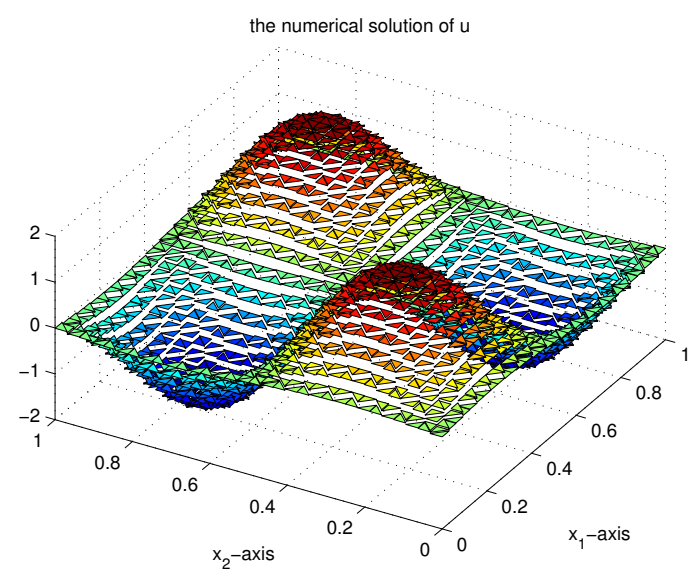

Figure 2. The numerical solution of $u$ with $h=\sqrt{2} \tau=\sqrt{2} / 40$ for Example 2 . 

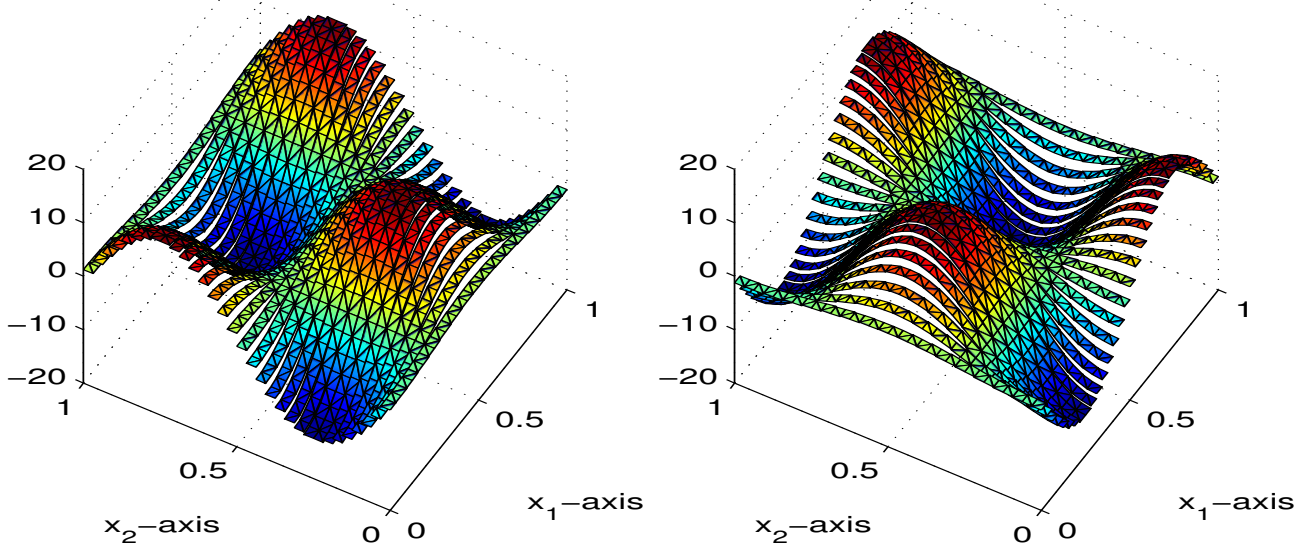

Figure 3. The numerical solution of $\sigma=\left(\sigma_{1}, \sigma_{2}\right)$ with $h=\sqrt{2} \tau=\sqrt{2} / 40$ for Example 2 .

Remark 2. When the diffusion coefficient $\varepsilon$ in Examples 1 and 2 is chosen to be 1/100, we do some numerical experiments with parameter $\alpha=0.1,0.3,0.5,0.7,0.9$. The numerical results show that the orders of convergence are similar to that of $\varepsilon=1$. The numerical results with $\varepsilon=1 / 100$ in Example $1(\alpha=0.1,0.3)$ and Example 2 $(\alpha=0.1)$ are given in Tables $8-10$, the other results with different parameter $\alpha$ are not a repeat description.

Table 8. Error results with $\alpha=0.1$ and $h=1 \times 10^{-4}$ for Example $1(\varepsilon=1 / 100)$.

\begin{tabular}{ccccccc}
\hline$\tau$ & $\|\boldsymbol{u}-\boldsymbol{U}\|_{L^{\infty}\left(L^{2}\right)}$ & Order & $\|\sigma-Z\|_{L^{\infty}\left(L^{2}\right)}$ & Order & $\|\sigma-Z\|_{L^{\infty}\left(H^{1}\right)}$ & Order \\
\hline $1 / 10$ & $3.2777 \times 10^{-4}$ & & $2.0606 \times 10^{-3}$ & & $1.3174 \times 10^{-2}$ & \\
$1 / 20$ & $9.5057 \times 10^{-5}$ & 1.7858 & $5.9811 \times 10^{-4}$ & 1.7846 & $3.8224 \times 10^{-3}$ & 1.7852 \\
$1 / 40$ & $2.7240 \times 10^{-5}$ & 1.8031 & $1.7192 \times 10^{-4}$ & 1.7987 & $1.0971 \times 10^{-3}$ & 1.8008 \\
$1 / 80$ & $7.7041 \times 10^{-6}$ & 1.8220 & $4.9147 \times 10^{-5}$ & 1.8066 & $3.1205 \times 10^{-4}$ & 1.8139 \\
\hline
\end{tabular}

Table 9. Error results with $\alpha=0.3$ and $h=1 \times 10^{-4}$ for Example $1(\varepsilon=1 / 100)$.

\begin{tabular}{ccccccc}
\hline$\tau$ & $\|\boldsymbol{u}-\boldsymbol{U}\|_{L^{\infty}\left(\boldsymbol{L}^{2}\right)}$ & Order & $\|\sigma-Z\|_{L^{\infty}\left(L^{2}\right)}$ & Order & $\|\sigma-Z\|_{L^{\infty}\left(H^{1}\right)}$ & Order \\
\hline $1 / 10$ & $1.6068 \times 10^{-3}$ & & $1.0098 \times 10^{-2}$ & & $6.4601 \times 10^{-2}$ & \\
$1 / 20$ & $5.1406 \times 10^{-4}$ & 1.6442 & $3.2311 \times 10^{-3}$ & 1.6439 & $2.0670 \times 10^{-2}$ & 1.6440 \\
$1 / 40$ & $1.6304 \times 10^{-4}$ & 1.6567 & $1.0253 \times 10^{-3}$ & 1.6560 & $6.5577 \times 10^{-3}$ & 1.6563 \\
$1 / 80$ & $5.1350 \times 10^{-5}$ & 1.6668 & $3.2341 \times 10^{-4}$ & 1.6646 & $2.0670 \times 10^{-3}$ & 1.6656 \\
\hline
\end{tabular}

Table 10. Error results with $\alpha=0.1$ and $h=\sqrt{2} \tau$ for Example $2(\varepsilon=1 / 100)$.

\begin{tabular}{ccccccc}
\hline $\boldsymbol{\tau}, \boldsymbol{h}$ & $\|\boldsymbol{u}-\boldsymbol{U}\|_{L^{\infty}\left(\mathbf{L}^{2}\right)}$ & Order & $\|\sigma-\mathrm{Z}\|_{L^{\infty}\left(\mathbf{L}^{2}\right)}$ & Order & $\|\sigma-\mathrm{Z}\|_{L^{\infty}(\boldsymbol{H}(\text { div }))}$ & Order \\
\hline $1 / 10, \sqrt{2} / 10$ & $2.5704 \times 10^{-1}$ & & 2.0136 & & $2.0549 \times 10^{1}$ & \\
$1 / 20, \sqrt{2} / 20$ & $1.3029 \times 10^{-1}$ & 0.9803 & 1.0081 & 0.9982 & $1.0348 \times 10^{1}$ & 0.9897 \\
$1 / 40, \sqrt{2} / 40$ & $6.5373 \times 10^{-2}$ & 0.9949 & $5.0378 \times 10^{-1}$ & 1.0007 & 5.1874 & 0.9963 \\
$1 / 80, \sqrt{2} / 80$ & $3.2715 \times 10^{-2}$ & 0.9987 & $2.5184 \times 10^{-1}$ & 1.0003 & 2.5955 & 0.9990 \\
\hline
\end{tabular}

\section{Conclusions}

We apply the MFVE method to treat the time-fractional reaction-diffusion equations. By introducing the auxiliary variable $\sigma$ and applying the L1-formula, we construct the MFVE scheme, prove the existence and uniqueness of the proposed scheme, and obtain the stability results which only depend on the source term function $f(X, t)$. We also obtain a priori error estimates for the variable $u$ (in $L^{2}(\Omega)$-norm) and the auxiliary variable $\sigma$ (in $\left(L^{2}(\Omega)\right)^{2}$-norm and $\boldsymbol{H}(\operatorname{div}, \Omega)$-norm) by using 
generalized MFVE projection and the properties of the transfer operator $\gamma_{h}$. Moreover, we briefly describe that the MFVE method can also be constructed in one-dimensional spatial regions, and give two numerical examples to examine the feasibility and effectiveness. In this article, we consider a linear model. In the future, we will try to study some nonlinear fractional models by using our method, which will be a challenge for us from the point of view of numerical analysis.

Author Contributions: All authors contributed to the draft of the manuscript, all authors read and approved the final manuscript.

Funding: This work was supported by the National Natural Science Foundation of China (11701299, 11761053, 11661058), the Natural Science Foundation of Inner Mongolia Autonomous Region (2016BS0105, 2017MS0107), the Program for Young Talents of Science and Technology in Universities of Inner Mongolia Autonomous Region (NJYT-17-A07), and the Prairie Talent Project of Inner Mongolia Autonomous Region.

Acknowledgments: The authors thank the anonymous referees and editors for their valuable suggestions and comments to improve this work.

Conflicts of Interest: The authors declare no conflict of interest.

\section{References}

1. Baleanu, D.; Güvenç, Z.B.; Machado J.A.T. New Trends in Nanotechnology and Fractional Calculus Applications; Springer: New York, NY, USA, 2010.

2. Baleanu, D.; Machado, J.A.T.; Luo, A. Fractional Dynamics and Control; Springer: New York, NY, USA, 2012.

3. Ortigueira. Fractional Calculus for Scientists and Engineers; Springer: New York, NY, USA, 2011.

4. Atanackovic, T.; Pilipovic, S.; Stankovic, B.; Zorica, D. Fractional Calculus with Applications in Mechanics: Vibrations and Diffusion Processes; Wiley-ISTE: London, UK, 2014.

5. Luchko Y. Wave-diffusion dualism of the neutral-fractional processes. J. Comput. Phys. 2015, 293, 40-52. [CrossRef]

6. Martínez-García, M.; Gordon, T.; Shu, L. Extended crossover model for human-control of fractional order plants. IEEE Access 2017, 5, 27622-27635. [CrossRef]

7. Diethelm, K. The Analysis of Fractional Differential Equations: An Application-Oriented Exposition Using Differential Operators of Caputo Type; Springer: Heidelberg, Germany, 2010.

8. Diethelm, K. Smoothness properties of solutions of Caputo-type fractional differential equations. Fract. Calc. Appl. Anal. 2007, 10, 151-160.

9. Carbotti, A.; Dipierro, S.; Valdinoci, E. Local Density of Solutions to Fractional Equations. De Gruyter Studies in Mathematics; De Gruyter: Berlin, Germany, 2019.

10. Metzler, R.; Klafter, J. Boundary value problems for fractional diffusion equations. Physica A 2000, 278, 107-125. [CrossRef]

11. Agrawal, O.P. A general solution for the fourth-order fractional diffusion-wave equation. Fract. Calc. Appl. Anal. 2000, 3, 1-12.

12. Arshad, S.; Baleanu, D.; Huang, J.F.; AI Qurashi, M.M.; Tang, Y.F.; Zhao, Y. Finite difference method for time-space fractional advection-diffusion equations with riesz derivative. Entropy 2018, 20, 321. [CrossRef]

13. Du, Y.W.; Liu, Y.; Li, H.; Fang, Z.C.; He, S. Local discontinuous Galerkin method for a nonlinear time-fractional fourth-order partial differential equation. J. Comput. Phys. 2017, 344, 108-126. [CrossRef]

14. Yuste, S.B. Weighted average finite difference methods for fractional diffusion equations. J. Comput. Phys. 2006, 216, 264-274. [CrossRef]

15. Yang, X.H.; Zhang, H.X.; Xu, D. Orthogonal spline collocation method for the two-dimensional fractional sub-diffusion equation. J. Comput. Phys. 2014, 256, 824-837. [CrossRef]

16. Li, C.P.; Ding, H.F. Higher order finite difference method for the reaction and anomalous-diffusion equation. Appl. Math. Model. 2014, 38, 3802-3821. [CrossRef]

17. Zhao, Y.; Bu, W.; Huang, J.; Liu, D.Y.; Tang, Y. Finite element method for two-dimensional space-fractional advection-dispersion equations. Appl. Math. Comput. 2015, 257, 553-565. [CrossRef]

18. Ford, N.J.; Xiao, J.Y.; Yan, Y.B. A finite element method for time fractional partial differential equations. Fract. Calcu. Appl. Anal. 2011, 14, 454-474. [CrossRef] 
19. Jin, B.T.; Lazarov, R.; Pasciak, J.; Zhou, Z. Error analysis of a finite element method for the space-fractional parabolic equation. SIAM J. Numer. Anal. 2014, 52, 2272-2294. [CrossRef]

20. Zheng, Y.Y.; Li, C.P.; Zhao, Z.G. A note on the finite element method for the space-fractional advection diffusion equation. Comput. Math. Appl. 2010, 59, 1718-1726. [CrossRef]

21. Liu, Y.; Du, Y.W.; Li, H.; Li, J.C.; He, S. A two-grid mixed finite element method for a nonlinear fourth-order reaction-diffusion problem with time-fractional derivative. Comput. Math. Appl. 2015, 70, 2474-2492. [CrossRef]

22. Liu, Y.; Zhang, M.; Li, H.; Li, J.C. High-order local discontinuous Galerkin method combined with WSGD-approximation for a fractional subdiffusion equation. Comput. Math. Appl. 2017, 73, 1298-1314. [CrossRef]

23. Wang, H.; Du, N. A superfast-preconditioned iterative method for steady-state space-fractional diffusion equations. J. Comput. Phys. 2013, 240, 49-57. [CrossRef]

24. Hejazi, H.; Moroney, T.; Liu, F. A finite volume method for solving the two-sided time-space fractional advection-dispersion equation. Cent. Eur. J. Phys. 2013, 11, 1275-1283. [CrossRef]

25. Hejazi, H.; Moroney, T.; Liu, F. Stability and convergence of a finite volume method for the space fractional advection-dispersion equation. J. Comput. Appl. Math. 2014, 255, 684-697. [CrossRef]

26. Zhuang, P.; Liu, F.; Turner, I.; Gu, Y.T. Finite volume and finite element methods for solving a one-dimensional space-fractional Boussinesq equation. Appl. Math. Model. 2014, 38, 3860-3870. [CrossRef]

27. Yang, Q.Q.; Turner, I.; Moroney, T.; Liu, F. A finite volume scheme with preconditioned Lanczos method for two-dimensional space-fractional reaction-diffusion equations. Appl. Math. Model. 2014, 38, 3755-3762. [CrossRef]

28. Cheng, A.J.; Wang, H.; Wang, K.X. A Eulerian-Lagrangian control volume method for solute transport with anomalous diffusion. Numer. Methods Part. Differ. Equ. 2015, 31, 253-267. [CrossRef]

29. Feng, L.B.; Zhuang, P.; Liu, F.; Turner, I. Stability and convergence of a new finite volume method for two-sided space-fractional diffusion equation. Appl. Math. Comput. 2015, 257, 52-65. [CrossRef]

30. Jia, J.H.; Wang, H. A preconditioned fast finite volume scheme for a fractional differential equation discretized on a locally refined composite mesh. J. Comput. Phys. 2015, 299, 842-862. [CrossRef]

31. Jia, J.H.; Wang, H. A fast finite volume method for conservative space-fractional diffusion equations in convex domains. J. Comput. Phys. 2016, 310, 63-84. [CrossRef]

32. Simmons, A.; Yang, Q.Q.; Moroney, T. A finite volume method for two-sided fractioanl diffusion equations on nonuniform meshes. J. Comput. Phys. 2017, 335, 747-759. [CrossRef]

33. Jiang, Y.J.; Xu, X.J.; A monotone finite volume method for time fractional Fokker-Planck equations. Sci. China Math. 2019, 62, 783-794. [CrossRef]

34. Karaa, S.; Mustapha, K.; Pani, A.K. Finite volume element method for two-dimensional fractional subdiffusion problems. IMA J. Numer. Anal. 2017, 37, 945-964. [CrossRef]

35. Karaa, S.; Pani, A.K. Error analysis of a FVEM for fractional order evolution equations with nonsmooth initial data. ESAIM Math. Model. Numer. Anal. 2018, 52, 773-801. [CrossRef]

36. Russell, T.F. Rigorous Block-Centered Discretizations on Irregular Grids: Improved Simulation of Complex Reservoir Systems; Technical Report No. 3, Project Report; Reservoir Simulation Research Corporation: Denver, CO, USA, 1995.

37. Chou, S.H.; Kwak, D.Y.; Vassilevski, P.S. Mixed covolume methods for the elliptic problems on triangular grids. SIAM J. Numer. Anal. 1998, 35, 1850-1861. [CrossRef]

38. Rui, H.X. Symmetric mixed covolume methods for parabolic problems. Numer. Methods Part. Differ. Equ. 2002, 18, 561-583. [CrossRef]

39. Yang, S.X.; Jiang, Z.W. Mixed covolume method for parabolic problems on triangular grids. Appl. Math. Comput. 2009, 215, 1251-1265. [CrossRef]

40. Fang, Z.C.; Li, H. An expanded mixed covolume method for Sobolev equation with convection term on triangular grids. Numer. Methods Part. Differ. Equ. 2013, 29, 1257-1277. [CrossRef]

41. Fang, Z.C.; Li, H. Numerical solutions to the regularized long wave equation based on mixed covolume method. Appl. Math. Mech. (Engl. Ed.) 2013, 34, 907-920. [CrossRef]

42. Arnold, D.N.; Douglas, J.; Gupta, C.P. A family of higher order mixed finite element methods for plane elasticity. Numer. Math. 1984, 45, 1-22. [CrossRef] 
43. Li, J.C. Full-order convergence of a mixed finite element method for fourth-order elliptic equations. J. Math. Anal. Appl. 1999, 230, 329-349. [CrossRef]

44. Brezzi, F.; Fortin, M. Mixed and Hybrid Finite Element Methods; Springer: New York, NY, USA, 1991.

45. Luo, Z.D. Mixed Finite Element Methods and Applications; Chinese Science Press: Beijing, China, 2006.

46. Li, R.H.; Chen, Z.Y.; Wu, W. Generalized Difference Methods for Differential Equations: Numerical Analysis of Finite Volume Methods; Marcel Dekker: New York, NY, USA, 2000.

47. Wang, X.; Li, Y.H. $L^{2}$ Error estimates for high order finite volume methods on triangular meshes. SIAM J. Numer. Anal. 2016, 54, 2729-2749. [CrossRef]

48. Chatzipantelidis, P.; Lazarov, R.D.; Thomée, V. Error estimates for a finite volume element method for parabolic equations in convex polygonal domains. Numer. Methods Part. Differ. Equ. 2004, 20, 650-674. [CrossRef]

49. Zhang, Z.Y. Error estimates of finite volume element method for the pollution in groundwater flow. Numer. Methods Part. Differ. Equ. 2010, 25, 259-274. [CrossRef]

50. Carstensen, C.; Dond, A.K.; Nataraj, N.; Pani, A.K. Three first-order finite volume element methods for Stokes equations under minimal regularity assumptions. SIAM J. Numer. Anal. 2018, 56, 2648-2671. [CrossRef]

51. Zhang, T.; Li, Z. An analysis of finite volume element method for solving the Signorini problem. Appl. Math. Comput. 2015, 270, 830-841. [CrossRef]

52. Lin, Y.M.; Xu, C.J. Finite difference/spectral approximations for the time-fractional diffusion equation. J. Comput. Phys. 2007, 225, 1533-1552. [CrossRef]

53. Sun, Z.Z.; Wu, X.N. A fully discrete scheme for a diffusion-wave system. Appl. Numer. Math. 2006, 56, 193-209. [CrossRef]

54. Adams, R. Sobolev Spaces; Academic Press: New York, NY, USA, 1975.

(C) 2019 by the authors. Licensee MDPI, Basel, Switzerland. This article is an open access article distributed under the terms and conditions of the Creative Commons Attribution (CC BY) license (http://creativecommons.org/licenses/by/4.0/). 